\title{
Intermolecular Multiple Quantum Coherences in Liquids
}

\author{
WOLFGANG RICHTER, ${ }^{1}$ WARREN S. WARREN ${ }^{2}$ \\ ${ }^{1}$ National Research Council, Institute for Biodiagnostics, Winnipeg, Manitoba, Canada \\ ${ }^{2}$ Princeton University, Princeton, New Jersey 08544
}

\begin{abstract}
In the early 1990s, the traditional framework of NMR spectroscopy was challenged through a series of simple experiments. The pulse sequences used consisted of a few RF pulses and a few gradient pulses, and the samples were mixtures of simple molecules. The spectra showed unexpected cross peaks between spins in different molecules. In order to explain these results, two basic assumptions had to be revisited: (1) the high-temperature approximation to the Boltzmann distribution at equilibrium, and (2) the cancellation of dipolar couplings in solution. A close look at the physics involved showed that correlations between spins in separate molecules exist even after a single pulse, and that dipolar couplings can make these correlations visible in the presence of gradient pulses. A comprehensive description of the effect is given here, and some present and future applications are discussed. (c) 2000 John Wiley \& Sons, Inc. Concepts Magn Reson 12: 396-409, 2000
\end{abstract}

KEY WORDS: high-temperature approximation; multiple quantum coherences; dipolar couplings; CRAZED; density matrix

\section{INTRODUCTION}

Since the first experiments in 1945 (1), NMR has arguably become the most versatile and broadly applicable form of spectroscopy. NMR is being successfully applied to solid, liquid, and gaseous media, to materials and living systems, and in the investigation of microscopic and macroscopic structures.

One reason for the enduring success of NMR is that the underlying physical phenomena are

Received 10 February 2000; revised 1 May 2000; accepted 5 May 2000.

Correspondence to: Dr. Wolfgang Richter

Concepts in Magnetic Resonance, Vol. 12(6) 396-409 (2000)

(C) 2000 John Wiley \& Sons, Inc. extremely well understood. This is especially so in the case of liquid-state high-resolution NMR spectroscopy. Transitions between nuclear spin states are virtually independent of other energy states of the molecules. Therefore, for example, the approximation of a two level system to a sole hydrogen nucleus in some molecule is indeed a very good one. Nobody seriously doubts that the response of a spin system to some sequence of radiofrequency pulses, delays, and gradients can be predicted with very high accuracy, even for pulse sequences consisting of thousands of RF pulses and delays. (The accuracy is not arbitrarily high, however, because some chaotic dynamics might occur, due to radiation damping or residual dipolar couplings (2).) If the spectrum that is produced by such a pulse sequence deviated from 
theoretical predictions, we would immediately assume that the spectrometer did not execute the pulse sequence properly.

Thus it was extraordinarily surprising when, in the early 1990s, a series of experiments was performed (3-4), whose results seemed to contradict conventional NMR theory. In these experiments, extremely simple pulse sequences (for example, two RF pulses and one or two gradient pulses, such as in the CRAZED sequence or the HOMOGENIZED sequence, which are discussed below) were applied to sometimes extremely simple spin systems (for example, a mixture of benzene and chloroform; each of these molecules contains only a single distinguishable proton). A shown in Figs. 1 and 2, the resulting spectra showed large peaks (no more than an order of magnitude below the peak corresponding to the full magnetization) at positions where there was no peak expected. The positions of these peaks correspond to those that would be produced by intermolecular multiple-quantum transitions, which had not been observed before in liquids, for a variety of perceived reasons, which will be discussed in detail below.

Several possible theories to explain these extra peaks were put forward at that time. A hint of the correct explanation is actually contained in a seemingly unrelated and much older paper (5); a recent article (6) describes this connection very nicely. In the experiment described in (5), a twopulse sequence is applied to a sample of solid Helium-3 in the presence of a constant magnetic field gradient; a similar experiment was later per-

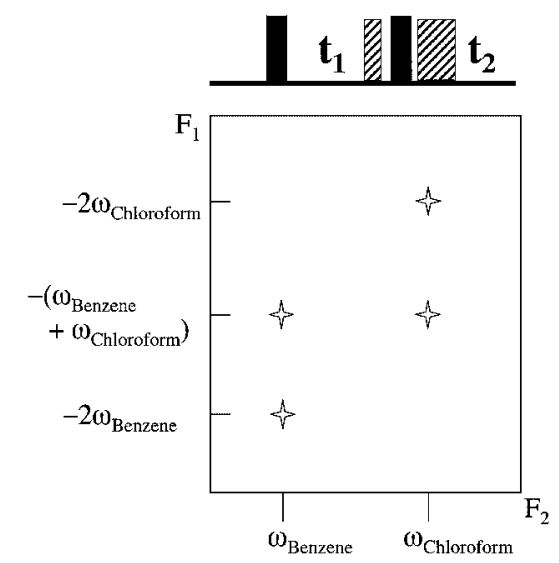

Figure 1 CRAZED spectrum of a mixture of benzene and chloroform. By conventional theory, this spectrum should be blank. Instead, there are peaks with all properties of intermolecular double-quantum peaks.

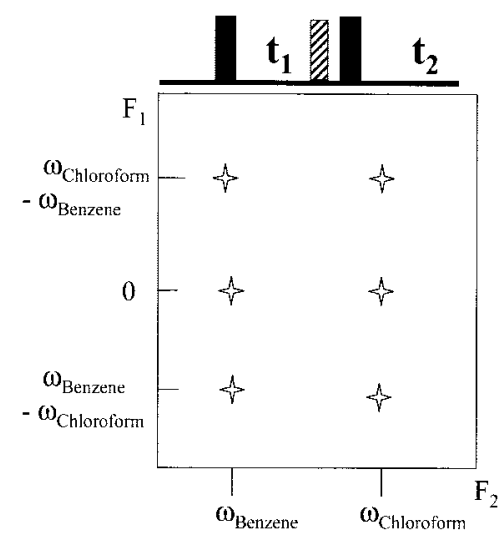

Figure 2 HOMOGENIZED spectrum of a mixture of benzene and chloroform. By conventional theory, this spectrum should be blank. Instead, there are peaks with all properties of intermolecular zero-quantum peaks.

formed, at much higher field, on a sample of water (7). This experiment produced a series of echoes in the free induction decay (FID). These echoes were explained through the concept of the "dipolar demagnetizing field." According to this explanation, the magnetic field gradient leads to the appearance of a dipolar field which makes the dynamics of the spin system nonlinear and therefore causes harmonics in the FID. This is the origin of a classical explanation of the extra peaks observed in 2D experiments. However, a quantum mechanical explanation was put forward as well. The classical explanation, while equally correct, lacks the predictive and intuitive power of the quantum mechanical one. For example, it is easy to see from the quantum mechanical picture why a pulse sequence like the HOMOGENIZED sequence (Fig. 2; see a brief discussion of this sequence at the end of this article) can produce narrow-line spectra in extremely inhomogeneous samples. A classical explanation of this is extremely awkward. In this article, therefore, we will focus on the quantum mechanical explanation.

Two assumptions that are explicitly or implicitly made in all textbooks have to be discarded. One of these assumptions concerns the equilibrium density operator for the spin system, and the other one concerns the dipolar interactions between spins. It turns out that these conventional assumptions predict experimental results correctly, as long as there are no gradient pulses in the pulse sequence. In the presence of gradient pulses, these assumptions have to be revisited, as explained below. It would also fail for a sample 
whose shape is far from spherical; this has been demonstrated experimentally by both Edsez (8) and Jeener (9).

\section{EARLY UNEXPECTED RESULTS: THE CRAZED EXPERIMENT}

First experimental results that seemed to contradict conventional theory were obtained in the early 1990s. Consider the prototypical 2D experiment, the correlation spectroscopy (COSY) experiment $(10,11)$. The pulse sequence for this experiment is shown in Fig. 3. A $\pi / 2$ pulse is applied to the sample, followed by an (incremented) evolution time $t_{1}$ and another $\pi / 2$ pulse. The subsequently measured FID is a function of two time variables, $t_{1}$ and $t_{2}$.

The quantum mechanical description of this pulse sequence is as follows. Let as assume there are two distinguishable, $J$-coupled spins in the sample, spin A and spin B. The first pulse excites single-quantum, single-spin coherences, such as $I_{x \mathrm{~A}}$. These evolve during $t_{1}$ under the scalar coupling between spins $\mathrm{A}$ and $\mathrm{B}$ into single-quantum two-spin coherences (such as $I_{y \mathrm{~A}} I_{z \mathrm{~B}}$ ), near the resonance frequency of spin $A\left(\omega_{A}\right)$. The second pulse converts them into other single-quantum two-spin coherences (such as $I_{z \mathrm{~A}} I_{y \mathrm{~B}}$ ), which then evolve, near $\omega_{B}$, into single-quantum, single-spin coherences, such as $I_{x \mathrm{~B}}$. Therefore, Fourier Transformation of the FID with respect to $t_{1}$ and $t_{2}$ yields a multiplet at $\omega_{\mathrm{A}}$ in $F_{1}$ and at $\omega_{\mathrm{B}}$ in $F_{2}$. For reasons of symmetry, there will also be three other sets of peaks at all possible combinations of $\omega_{\mathrm{A}}$ and $\omega_{\mathrm{B}}$, as shown in Fig. 4.

Consider now a COSY experiment with a sample containing two compounds with one spin species each, for example, a mixture of acetone and benzene. Of course, the six equivalent spins in each molecule are scalar-coupled ( $J$ coupled) to one another. It can, however, be rigorously shown that this coupling can never be observed in any conceivable NMR experiment (12), (unless, for example, one of the carbons is a C-13, which would make some of the protons inequivalent.

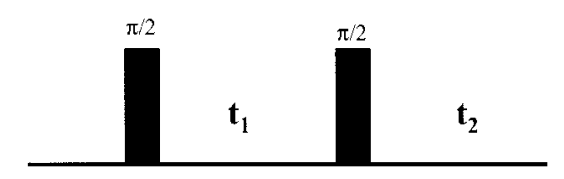

Figure 3 COSY pulse sequence.

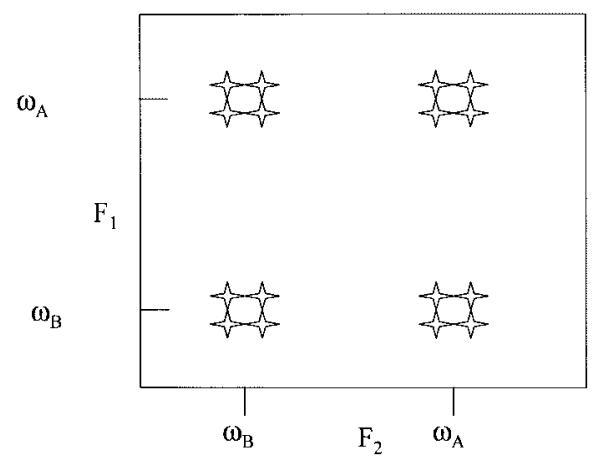

Figure 4 COSY spectrum of a molecule with two coupled spins. Note that there are cross peaks between all lines.

Hence the 1D (single pulse) spectrum of this sample contains two lines only. The COSY spectrum, as shown schematically in Fig. 5, consists of the two diagonal peaks only. There are no cross peaks because the corresponding spins are in separate molecules and are not $J$ coupled to one another. This, of course, is the power of the COSY experiment-to identify spins that are $J$ coupled.

\section{Gradient Filters}

Now let us modify this experiment in a seemingly meaningless way. We will add a 'double-quantum gradient filter,' which lets only a double-quantum signal be converted into magnetization. This filter is based on the fact that a gradient pulse modulates the resonance frequency of the spins as a linear function of space along the gradient axis; hence, classically speaking, transverse magnetization is wound up into a helix by such a pulse. Note that this helix represents a highly ordered

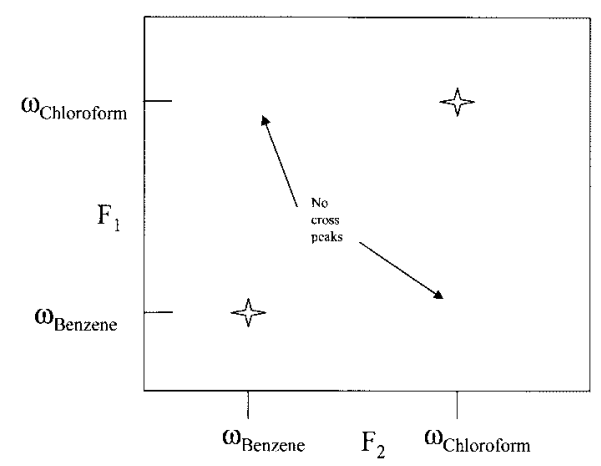

Figure 5 COSY spectrum of a sample containing acetone and benzene. There are no cross peaks because there is no intermolecular coupling. 
state, which has, however, no net magnetization associated with it. This helix can subsequently be unwound by another gradient pulse of opposite polarity. This classical picture can be extended to multiple-quantum coherences (there is no helix of a measurable quantity then, but the analogy is valid). If a gradient pulse is applied to a doublequantum coherence, which naturally evolves at twice the basic resonance frequency, the (virtual) helix that is created has only half the pitch of the helix created by the same gradient applied to a single-quantum coherence. In other words, the double-quantum helix is twice as tightly wound up as the single-quantum helix. It follows that a pair of gradient pulses whose areas are at a ratio of $1: 2$ acts as a double-quantum filter - a quantity that is magnetization after this filter has to have been a double-quantum coherence during the first gradient pulse; then and only then would the second gradient pulse exactly unwind the helix and create net magnetization. This also means that, if there is magnetization after this filter, a 2-quantum to 1-quantum transformation must have taken place between the two gradient pulses.

We will now add a double-quantum gradient filter at the second pulse of the COSY experiment, as shown in Fig. $6 . G$ is the amplitude of the first gradient, and $T$ is its duration; the area of the second gradient pulse is twice that of the first one. It is easy to see that there is no signal expected after this pulse sequence. As mentioned above, the first pulse of the COSY experiment creates single-quantum coherences, which will not pass the double-quantum filter. Hence, according to conventional NMR theory and prior to 1990 , no one would have expected a signal from this pulse sequence, save for imperfections in the pulses, and effects caused by relaxation. This is why this pulse sequence was called the CRAZED (COSY Revamped by Asymmetric Z-Gradient Echo Detection) sequence. However, in (2) it was shown that there is a large signal after this pulse sequence. Peaks appear both on the pseudo-diagonal $F_{1}=-2 F_{2}$, and as cross peaks between benzene and acetone protons (Fig. 1).

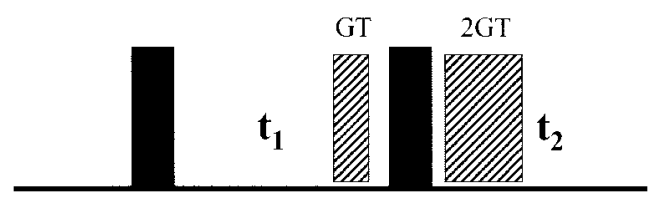

Figure 6 CRAZED sequence.
What does it mean that there is magnetization at the end of the gradient filter? It can only be so if two conditions are true: (1) There must have been a double-quantum coherence evolving before the gradient filter; and (2) this coherence must have been transformed into a single-quantum coherence after the first gradient pulse. This, in turn, implies two things: (1) A single pulse must create double-quantum coherences; and (2) there must be a net coupling between benzene and acetone protons. Both these statements seem to contradict conventional NMR theory, and we will discuss them now.

\section{THE DENSITY OPERATOR}

The density operator, commonly designated as $\rho$, describes the state of a spin system at any point during the pulse sequence. For a comprehensive discussion of the density operator, see (13).

At thermal equilibrium, the spin system follows a Boltzmann distribution. The density operator is given by

$$
\rho_{\mathrm{eq}}=\frac{\exp (-\mathscr{H} \hbar / k T)}{\operatorname{tr}[\exp (-\mathscr{H} \hbar / k T)]}
$$

Note that we used the Hamiltonian operator, $\mathscr{H}$, in units of angular frequency. This is often convenient in spectroscopic applications; the conventional Hamiltonian (energy) operator can be obtained by multiplying by $\hbar$.

Let us discuss some examples. The simplest one is given by an isolated spin $\frac{1}{2}$, such as hydrogen, which possesses two energy levels in a magnetic field. The Hamiltonian of this system is given by

$$
\mathscr{H}=\omega I_{z}
$$

where $I_{z}$ is the unitless angular momentum operator, which here represents the action of the static magnetic field, and $\omega$ is the Larmor frequency. A convenient basis set is given by the two basis function $\alpha=|1 / 2,1 / 2\rangle$ and $\beta=$ $|1 / 2,-1 / 2\rangle$. The matrix elements of $I_{z}$ can be computed by

$$
I_{z}=\left(\begin{array}{ll}
\left\langle\beta\left|I_{z}\right| \beta\right\rangle & \left\langle\beta\left|I_{z}\right| \alpha\right\rangle \\
\left\langle\alpha\left|I_{z}\right| \beta\right\rangle & \left\langle\alpha\left|I_{z}\right| \alpha\right\rangle
\end{array}\right)=\frac{1}{2}\left(\begin{array}{rr}
-1 & 0 \\
0 & +1
\end{array}\right)
$$


At thermal equilibrium we have for this spin,

$$
\begin{aligned}
\rho_{0} & =\frac{\exp (-\mathscr{H} / k T)}{\operatorname{tr}[\exp (-\mathscr{H} / k T)]}=\frac{\exp \left((-\hbar \omega / k T) I_{z}\right)}{\operatorname{tr}\left[\exp \left((-\hbar \omega / k T) I_{z}\right)\right]} \\
\left(\begin{array}{cc}
\exp \left(+\frac{\hbar \omega}{2 k T}\right) & 0 \\
0 & \exp \left(-\frac{\hbar \omega}{2 k T}\right)
\end{array}\right) & \frac{(\hbar \omega / 2 k T))}{\exp (+(\hbar \omega / 2 k T))+\exp (-(\hbar)}
\end{aligned}
$$

The equilibrium population of the two states is given by the diagonal elements of this matrix. Therefore, the ratio of the population of the two states is

$$
\frac{P_{\beta}}{P_{\alpha}}=\frac{\exp (+\hbar \omega / 2 k T)}{\exp (-\hbar \omega / 2 k T)}=\exp \left(+\frac{\hbar \omega}{k T}\right)
$$

An explicit calculation for a $600 \mathrm{MHz}$ machine $\left(\omega=600 \mathrm{MHz}^{*} 2 \pi=3.769^{*} 10^{9} \mathrm{~s}^{-1}\right)$ and room temperature yields $P_{\beta} / P_{\alpha}=1.0001$.

Now suppose we have two isolated spins in the system. Accordingly, there are four energy levels, $\alpha_{1} \alpha_{2}, \alpha_{1} \beta_{2}, \beta_{1} \alpha_{2}$, and $\beta_{1} \beta_{2}$, and the density operator describing this spin system is represented by a four by four matrix,

$$
\begin{aligned}
& I_{z 1}=\left(\begin{array}{ccc}
\left\langle\beta_{1} \beta_{2}\left|I_{z 1}\right| \beta_{1} \beta_{2}\right\rangle & \bullet & \bullet \\
\bullet & \left\langle\alpha_{1} \beta_{2}\left|I_{z 1}\right| \alpha_{1} \beta_{2}\right\rangle & \bullet \bullet \\
\bullet & \vdots & \bullet
\end{array}\right) \\
& =\frac{1}{2}\left(\begin{array}{rrrr}
-1 & 0 & 0 & 0 \\
0 & -1 & 0 & 0 \\
0 & 0 & 1 & 0 \\
0 & 0 & 0 & 1
\end{array}\right) \\
& I_{z 1}+I_{z 2}=\frac{1}{2}\left(\begin{array}{rrrr}
-1 & 0 & 0 & 0 \\
0 & -1 & 0 & 0 \\
0 & 0 & +1 & 0 \\
0 & 0 & 0 & +1
\end{array}\right) \\
& +\frac{1}{2}\left(\begin{array}{rrrr}
-1 & 0 & 0 & 0 \\
0 & +1 & 0 & 0 \\
0 & 0 & -1 & 0 \\
0 & 0 & 0 & +1
\end{array}\right) \\
& =\left(\begin{array}{rrrr}
-1 & 0 & 0 & 0 \\
0 & 0 & 0 & 0 \\
0 & 0 & 0 & 0 \\
0 & 0 & 0 & +1
\end{array}\right)
\end{aligned}
$$

The population ratio between lowest and highest energy states is therefore

$$
P_{\beta \beta} / P_{\alpha \alpha}=\exp \left(\frac{2 \hbar \omega}{k T}\right)=1.0002
$$

Similarly, we can calculate the populations for a large number of spins. For example, for a system containing $10^{4}$ spins (which would, incidentally, still be a microscopic sample), we find, for the ratio of the population of the lowest energy state and of the highest energy state,

$$
P_{\beta \ldots \beta} / P_{\alpha \cdots \alpha}=\exp \left(\frac{10^{4} \hbar \omega}{k T}\right)=2.6
$$

Compare these results to the traditional 'hightemperature' approximation to the equilibrium density operator. In that approximation, the exponential is expanded in a truncated Taylor series, as follows:

$$
\begin{aligned}
\rho_{0}^{\mathrm{HT}} & =\frac{\exp \left((-\hbar \omega / k T) \sum_{i} I_{z i}\right)}{\operatorname{tr}[\exp (-\mathscr{H} / k T)]} \\
& \approx \frac{\mathbf{1}-(\hbar \omega / k T) \sum_{i} I_{z i}}{\operatorname{tr}[\exp (-\mathscr{H} / k T)]}
\end{aligned}
$$

This is very convenient, because we can start any pulse sequence calculation with an equilibrium state of

$$
\rho_{\mathrm{eq}} \propto I_{z i}
$$

because $I_{z}$ is the only variable that evolves in time. What are we missing by this approximation? The argument that is commonly given for the validity of the approximation is that the Boltzmann factor is a small number $\left(\hbar \omega / k T<10^{-4}\right)$, and higher order terms in this expansion are therefore negligible.

This is a fallacy. If the Taylor expansion were not truncated, it would continue as

$$
\begin{aligned}
\rho_{0}= & \frac{\exp \left((-\hbar \omega / k T) \sum_{i} I_{z i}\right)}{\operatorname{tr}[\exp (-\mathscr{H} / k T)]} \\
& \mathbf{1}-(\hbar \omega / k T) \sum_{i} I_{z i} \\
\approx & \frac{+(\hbar \omega / k T)^{2} \sum_{i} \sum_{j} I_{z i} I_{z j}-\cdots+\cdots}{\operatorname{tr}[\exp ((-\mathscr{H} / k T))]}
\end{aligned}
$$

and, even though the quadratic term has a coefficient that is several orders of magnitude smaller 
than that of the linear term, the double sum in the quadratic term has $N$ times as many members as the single sum in the linear term. For a typical NMR sample, $N=10^{20}$ and $N^{2}=10^{40}$; hence it is not at all obvious that this expansion even converges!

Let us now calculate the populations of the states that are predicted by the high-temperature approximation. For a two-spin system, for example, we find that

$$
\begin{aligned}
& 1-\frac{\hbar \omega}{k T}\left(I_{z 1}+I_{z 2}\right) \\
& \quad=\left(\begin{array}{cccc}
1+\frac{\hbar \omega}{k T} & 0 & 0 & 0 \\
0 & 1 & 0 & 0 \\
0 & 0 & 1 & 0 \\
0 & 0 & 0 & 1-\frac{\hbar \omega}{k T}
\end{array}\right)
\end{aligned}
$$

which means that the predicted ratio of populations of the highest and lowest energy levels is

$$
P_{\beta \beta} / P_{\alpha \alpha}=\frac{1+(\hbar \omega / k T)}{1-(\hbar \omega / k T)}=1.0002
$$

in accordance with the exact calculation. However, for $10^{4}$ spins, we find

$$
P_{\alpha \cdots \alpha} / P_{\beta} \ldots \beta=\frac{1+5000(\hbar \omega / k T)}{1-5000(\hbar \omega / k T)}=2.9
$$

which is significantly different from the exact result of 2.6 obtained above (Eq. [9]). For larger number of spins, the two results diverge even more. The reason for this is, of course, that the approximation made by the truncated Taylor series, $\exp (x) \approx 1+x$, only holds if $x$ is small compared to 1 . Hence the high-temperature approximation fails to predict the population of the energy levels appropriately for any macroscopic sample.

It is possible to express the exact density operator in closed form as a product of individual spin operators (14). However, for the purpose of this article, we will keep the usual expansion and terminate it after the quadratic term. This affords the necessary insight into the CRAZED experi- ment; a generalization to other orders of coherence is conceptually straightforward and may be found in the primary literature (14).

\section{DipOLAR COUPLINGS}

The classical dipolar interaction energy between two magnetic dipoles 1 and 2 is given by

$$
E_{\mathrm{dip}}=\frac{\boldsymbol{\mu}_{0}}{4 \pi} \frac{1}{\left|\mathbf{r}_{12}\right|^{3}}\left[\boldsymbol{\mu}_{1} \cdot \boldsymbol{\mu}_{2}-3 \frac{\left(\boldsymbol{\mu}_{1} \cdot \mathbf{r}_{12}\right)\left(\boldsymbol{\mu}_{2} \cdot \mathbf{r}_{12}\right)}{\left|\mathbf{r}_{12}\right|^{2}}\right]
$$

where the $\boldsymbol{\mu}_{i}$ are the magnetic moments, and $\mathbf{r}_{i j}$ is the vector connecting them.

The dipolar Hamiltonian is constructed in full analogy to this, replacing the classical magnetic moment by its quantum mechanical analogue (15):

$$
\begin{aligned}
\mathscr{H}_{d}= & \frac{\mu_{0}}{4 \pi} \sum_{k>l} \frac{\gamma_{k} \gamma_{l} \hbar}{r_{k l}^{3}} \\
& \times\left(\mathbf{I}_{k} \mathbf{I}_{l}-3 \frac{\left(\mathbf{I}_{k} \cdot \mathbf{r}_{k l}\right)\left(\mathbf{I}_{l} \cdot \mathbf{r}_{k l}\right)}{r_{k l}^{2}}\right)
\end{aligned}
$$

Here, the $\gamma_{i}$ are the gyromagnetic ratios of the respective nuclei. The Hamiltonian operator is, again, given in frequency units. This can be rewritten in polar coordinates; we can take also take into account that nonsecular terms vanish because of the Zeeman interaction (10). The remaining dipolar Hamiltonian is

$$
\begin{aligned}
H_{d}^{\mathrm{sec}}= & \frac{\mu_{0}}{4 \pi} \sum_{k=0}^{N} \sum_{l=0}^{N} \frac{\gamma_{k} \gamma_{l} \hbar}{4 r_{k l}^{3}} \\
& \times\left(1-3 \cos ^{2} \vartheta_{k l}\right)\left(3 I_{k z} I_{l z}-\mathbf{I}_{k} \cdot \mathbf{I}_{l}\right) \\
= & \sum_{k=0}^{N} \sum_{l=0}^{N} D_{k l}\left(3 I_{k z} I_{l z}-\mathbf{I}_{k} \cdot \mathbf{I}_{l}\right) \\
D_{k l} & \equiv \frac{\mu_{0}}{4 \pi} \frac{\gamma_{k} \gamma_{l} \hbar}{4 r_{k l}^{3}}\left(1-3 \cos ^{2} \vartheta_{k l}\right)
\end{aligned}
$$

In this equation, $\theta_{k l}$ is the angle between the internuclear vector and the main magnetic field. We have also defined the 'dipolar coupling constant,' $D_{k l}$. Note that the double sum is unre- 
stricted; hence each spin pair is counted twice. This is included in the definition of $D_{k l}$.

What is the magnitude of the dipolar couplings? For convenience, we will first calculate the value of the dipolar coupling constant (for two protons),

$$
\begin{aligned}
D_{k l} & \equiv \frac{\mu_{0}}{4 \pi} \frac{\gamma_{k} \gamma_{l} \hbar}{4 r_{k l}^{3}}\left(1-3 \cos ^{2} \vartheta_{k l}\right) \\
& =188.7 \frac{\mathrm{rad}}{s} *\left(\frac{1-3 \cos ^{2} \vartheta_{k l}}{\left(r_{k l} / n m\right)^{3}}\right)
\end{aligned}
$$

For example, for a distance of $5 \AA(0.5 \mathrm{~nm})$, and $\theta=90^{\circ}$, we find $D_{k l}=3020 \mathrm{rad} \mathrm{s}^{-1}$, or approximately $480 \mathrm{~Hz}$ !

The question arises then why we see sharp lines (less than $1 \mathrm{~Hz}$ in a well-shimmed sample) at all in the NMR spectrum of a liquid. If the dipolar couplings are on the order of hundreds of $\mathrm{Hz}$ or more for nearby protons, then the spectral lines should be just that wide, and we would not see any structure in the spectrum. This is, of course, precisely the case for a solid.

There are indeed two separate mechanisms that produce narrow lines in liquids. One mechanism applies to pairs of nearby spins, and one applies to spins far apart. Note that, over the surface of an isotropic sphere, the dipolar interactions average to zero because

$$
\int_{\vartheta=0}^{\pi} \int_{\varphi=0}^{2 \pi}\left[\left(3 \cos ^{2} \vartheta-1\right) \sin \vartheta d \vartheta d \varphi\right]=0
$$

(The extra factor of $\sin \theta$ comes from the fact that the number of surface elements on a sphere is proportional to $\sin \theta$.)

The two mechanisms that average out dipolar couplings are the following.

(1) Short range dipolar interactions average to zero through diffusion. For a back-of-the-envelope calculation, let us assume that the diffusion coefficient of the liquid is $D=2.3 \times 10^{-9} \mathrm{~m}^{2} / \mathrm{s}$ (this is the diffusion coefficient of water at room temperature). The root mean square distance that a molecule diffuses in a given time $t$ in some direction is

$$
r_{\mathrm{rms}}=\sqrt{2 D t}
$$

The effects of individual couplings can be ignored if they only remain unaltered for a time such that

$$
\begin{aligned}
& D_{k l} t \ll 1 \\
& D_{k l} t=188.7 \frac{\mathrm{rad}}{s} *\left(\frac{1-3 \cos ^{2} \vartheta_{k l}}{\left(r_{k l} / n m\right)^{3}}\right) * \frac{r_{\mathrm{rms}}^{2}}{2 D}
\end{aligned}
$$

For our estimation, let us assume that the full range of angles is sampled when the spin moves through a distance of the order of the intermolecular separation. Then, for two spins at the closest possible distance, say $0.2 \mathrm{~nm}$, we find

$$
\begin{aligned}
D_{k l} t & =188.7 \frac{\mathrm{rad}}{s} *\left(\frac{3}{0.2^{3}}\right) * \frac{\left(0.2 * 10^{-9}\right)^{2}}{2 D} \\
& =6.2 * 10^{-7}
\end{aligned}
$$

This is indeed much smaller than 1 . If we move the spins farther apart, that number decreases even further, as it is approximately inversely proportional to the separation distance. This means that all individual spin-spin dipolar interactions can be ignored.

(2) Long-range dipolar interactions are averaged out by magnetic isotropy. For long range dipolar interactions, the averaging has to be considered somewhat differently. Here we have to look at a large number of virtually constant interactions simultaneously (each individual spin pair does not sample the full range of angles any more, but is virtually static on an NMR time scale. We will have to add up all individual interactions, with a proper weighting for each spin pair. If the liquid is magnetically isotropic (that is, if the magnetization is the same anywhere in the sample), dipolar interactions add up to zero by virtue of spherical symmetry. It is a fallacy to conclude that long range dipolar interactions are negligible because of the $r^{-3}$ dependence, because the number of spins at a given distance increases as $r^{2}$. The sum of all dipolar interactions at a given spin only falls off as $r^{-1}$.

Therefore, we will have to explicitly consider long-range dipolar interactions whenever the magnetization of the sample is a function of location. This happens whenever gradient pulses are applied during the pulse sequence. Dipolar interactions will also reappear whenever the sample is not spherical. However, in the absence of gradients, the effects of these couplings are usually 
masked by radiation damping and will not be considered here.

\section{QUANTITATIVE ANALYSIS OF THE CRAZED EXPERIMENT}

\subsection{Overview}

Considering the discussion of Sections 3 and 4, we can see now how the strange peaks in the CRAZED spectrum originate. The pulse sequence was given in Fig. 6. The quadratic term in the equilibrium density operator contains two-spin terms such as $I_{z 1} I_{z 2}$. A $\pi / 2$ pulse transforms this into $I_{x 1} I_{x 2}$, which is actually a mixture of doubleand zero-quantum coherences. Hence doublequantum coherences evolve during $t_{1}$; they eventually give rise to the cross peaks. They also pass the double-quantum gradient filter, because the second $\pi / 2$ pulse transforms a term such as $I_{x 1} I_{y 2}$ into $I_{z 1} I_{y 2}$, which is a single-quantum, two-spin term. By then, the sample exhibits anisotropic magnetization (because of the gradient pulses), and dipolar couplings reappear. Therefore, dipolar couplings between spins 1 and 2 transform $-I_{z 1} I_{y 2}$ into $I_{x 2}$, which is magnetization. In Fig. 7, this is depicted schematically.

\subsection{Quantitative Analysis}

We start out the density matrix calculation with the expansion terminated after the quadratic term,

$$
\rho=1-\frac{\hbar \omega}{k T} \sum_{i=1}^{N} I_{z i}+\frac{1}{2}\left(\frac{\hbar \omega}{k T}\right)^{2} \sum_{i=1}^{N} \sum_{j=1}^{N} I_{z i} I_{z j}
$$

We can immediately simplify this, considering that only the second order term will survive the

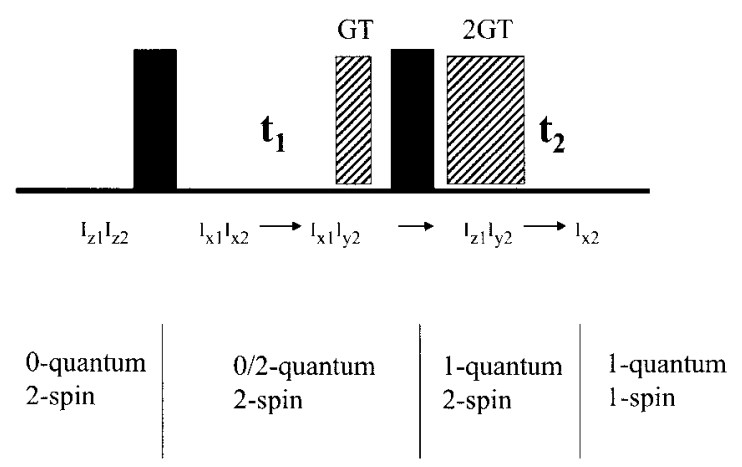

Figure 7 Evolution of some relevant spin operators during the CRAZED sequence. double-quantum filter (we already know that the high-temperature density matrix cannot contribute to the signal of the CRAZED sequence, as discussed in Sect. 2). Furthermore, we will leave out all normalizing constants; at the end, we will compare the signal to that after a single pulse, which contains the same constants. Therefore we will start with

$$
\rho=\frac{1}{2}\left(\frac{\hbar \omega}{k T}\right)^{2} \sum_{i=1}^{N} \sum_{j=1}^{N} I_{z i} I_{z j}
$$

Now we apply the first pulse. This transforms the density operator into

$$
\rho=\frac{1}{2}\left(\frac{\hbar \omega}{k T}\right)^{2} \sum_{i=1}^{N} \sum_{j=1}^{N} I_{x i} I_{x j}
$$

This term now contains a mixture of zero-quantum operators and double-quantum operators. During the first time interval $\left(t_{1}\right)$, then the system evolves into

$$
\begin{aligned}
\rho= & \frac{1}{2}\left(\frac{\hbar \omega}{k T}\right)^{2} \sum_{i=1}^{N} \sum_{j=1}^{N}\left[I_{x i} \cos \left(\omega t_{1}\right)+I_{y i} \sin \left(\omega t_{1}\right)\right] \\
& \times\left[I_{x j} \cos \left(\omega t_{1}\right)+I_{y j} \sin \left(\omega t_{1}\right)\right]
\end{aligned}
$$

The gradient pulse adds a space-dependent evolution frequency of magnitude $\gamma G s_{i}$ to the normal evolution frequency of $\Delta \omega$, where $s_{i}$ is the location of spin $i$ along the gradient axis. As discussed above, net dipolar couplings are reintroduced with the first gradient pulse; for simplicity, however, we will begin to consider dipolar couplings only at the end of the second gradient pulse. This is appropriate because the gradient pulses are short (in the common implementations they are on the order of a few milliseconds). The density operator at the end of $t_{1}$ (after the gradient) is therefore

$$
\begin{aligned}
\rho= & \frac{1}{2}\left(\frac{\hbar \omega}{k T}\right)^{2} \\
& \times \sum_{i=1}^{N} \sum_{j=1}^{N}\left[I_{x i} \cos \left(\omega t_{1}+\gamma G T s_{i}\right)\right. \\
& \left.+I_{y i} \sin \left(\omega t_{1}+\gamma G T s_{i}\right)\right] \\
& \times\left[I_{x j} \cos \left(\omega t_{1}+\gamma G T s_{j}\right)\right. \\
& \left.+I_{y j} \sin \left(\omega t_{1}+\gamma G T s_{j}\right)\right]
\end{aligned}
$$


The second $90^{\circ}$ pulse then transforms the density operator into

$$
\begin{aligned}
\rho= & \frac{1}{2}\left(\frac{\hbar \omega}{k T}\right)^{2} \\
& \times \sum_{i=1}^{N} \sum_{j=1}^{N}\left[-I_{z i} \cos \left(\omega t_{1}+\gamma G T s_{i}\right)\right. \\
& \left.+I_{y i} \sin \left(\omega t_{1}+\gamma G T s_{i}\right)\right] \\
& \times\left[-I_{z j} \cos \left(\omega t_{1}+\gamma G T s_{j}\right)\right. \\
& \left.+I_{y j} \sin \left(\omega t_{1}+\gamma G T s_{j}\right)\right]
\end{aligned}
$$

Now we can simplify this equation. Remember that no more RF pulses follow, and that only RF pulses can change the number of quanta in an operator (the dipolar couplings only contain zero-quantum operators). Therefore, we only have to consider single-quantum terms, that is, terms with a single transverse operator from now on. Those terms are,

$$
\begin{aligned}
\rho= & \left(\frac{\hbar \omega}{k T}\right)^{2} \sum_{i=1}^{N} \sum_{j=1}^{N}\left[-I_{z i} \cos \left(\omega t_{1}+\gamma G T s_{i}\right)\right. \\
& \left.\times I_{y j} \sin \left(\omega t_{1}+\gamma G T s_{j}\right)\right]
\end{aligned}
$$

The factor of $\frac{1}{2}$ was deleted, because we undercounted the terms in Eq. [31] by a factor of 2 .

During the second gradient pulse, this evolves into

$$
\begin{aligned}
\rho=\left(\frac{\hbar \omega}{k T}\right)^{2} \sum_{i=1}^{N} \sum_{j=1}^{N}-I_{z i} \cos \left(\omega t_{1}+\gamma G T s_{i}\right) \\
\\
\times\left[I_{y j} \sin \left(\omega t_{1}+\gamma G T s_{j}\right) \cos \left(2 \gamma G T s_{j}\right)\right. \\
\left.-I_{x j} \sin \left(\omega t_{1}+\gamma G T s_{j}\right) \sin \left(2 \gamma G T s_{j}\right)\right]
\end{aligned}
$$

Note that we have, for convenience, disregarded the chemical shift evolution during the second gradient pulse.

We can transform the products of the trigonometric functions into sums, using the usual trigonometric identities. We use

$\cos A \sin B \cos C$

$$
\begin{aligned}
& =\frac{1}{4}[\sin (A+B+C)+\sin (A+B-C) \\
& -\sin (A-B-C)-\sin (A-B+C)]
\end{aligned}
$$

$\cos A \sin B \sin C$

$$
\begin{aligned}
& =\frac{1}{4}[\cos (A-B+C)+\cos (A+B-C) \\
& -\cos (A+B+C)-\cos (A-B-C)]
\end{aligned}
$$

Now consider the following simplification:

A function that depends on the absolute position of the spin in the sample will average to zero. For example, if a term depends on $\sin \left(\gamma G T s_{i}\right)$, then it will average to zero if the gradient pulse winds up a helix with many turns over the sample. The only terms that do not average to zero in this manner are the ones that depend solely on a difference in position, which are the terms $\sin (A$ $+B-C)$ and $\cos (A+B-C)$ in the above equation. Hence we obtain

$$
\begin{aligned}
\rho= & \frac{1}{4}\left(\frac{\hbar \omega}{k T}\right)^{2} \\
& \times \sum_{i=1}^{N} \sum_{j=1}^{N}\left\{-I_{z i} I_{y j} \cos \left[2 \omega t_{1}+\gamma G T\left(s_{i}-s_{j}\right)\right]\right. \\
& \left.+I_{z i} I_{x j} \sin \left[2 \omega t_{1}+\gamma G T\left(s_{i}-s_{j}\right)\right]\right\}
\end{aligned}
$$

We can further simplify this expression by using

$$
\begin{aligned}
& \cos (A+B)=\cos A \cos B-\sin A \sin B \\
& \sin (A+B)=\sin A \cos B+\cos A \sin B
\end{aligned}
$$

Note that for every spin pair ' $i j$,' there is another interaction ' $j i$ ' in the sample. Since the sine is an odd function, the terms containing $\sin B$ in the above equation vanish. Hence we are left with

$$
\begin{aligned}
\rho= & \frac{1}{4}\left(\frac{\hbar \omega}{k T}\right)^{2} \\
& \times \sum_{i=1}^{N} \sum_{j=1}^{N}\left\{-I_{z i} I_{y j} \cos \left(2 \omega t_{1}\right)\right. \\
& +\cos \left[\gamma G T\left(s_{i}-s_{j}\right)\right] \\
& \left.+I_{z i} I_{x j} \sin \left(2 \omega t_{1}\right) \cos \left[\gamma G T\left(s_{i}-s_{j}\right)\right]\right\} \\
= & \frac{1}{4}\left(\frac{\hbar \omega}{k T}\right)^{2} \\
& \times \sum_{i=1}^{N} \sum_{j=1}^{N}\left\{\left[I_{z i} I_{x j} \cos \left(2 \omega t_{1}\right)\right.\right. \\
& \left.-I_{z i} I_{y j} \sin \left(2 \omega t_{1}\right)\right] \\
& \left.\times \cos \left[\gamma G T\left(s_{i}-s_{j}\right)\right]\right\}
\end{aligned}
$$


Now we let this evolve during $t_{2}$, finally using dipolar couplings. It can be shown that the only part of the dipolar Hamiltonian that matters here is the longitudinal part (11). Evolution under this Hamiltonian takes place, for example, as

$$
\begin{aligned}
2 I_{z 1} I_{x 2} \stackrel{D_{12} I_{z 1} I_{z 2} t}{\longrightarrow} & 2 I_{z 1} I_{x 2} \\
& \times \cos \left(D_{i j} t\right)+I_{y 2} \sin \left(D_{i j} t\right)
\end{aligned}
$$

In our case, we find

$$
\text { (36) } \begin{aligned}
\stackrel{\sum \sum 3 D_{i j} I_{z i} I_{z j} t_{2}}{\longrightarrow} & \frac{3}{8}\left(\frac{\hbar \omega}{k T}\right)^{2} \\
& \times \sum_{i=1}^{N} \sum_{j=1}^{N}\left[I_{y j} \cos \left(2 \omega t_{1}\right)\right. \\
& \left.+I_{x j} \sin \left(2 \omega t_{1}\right)\right] \\
& \times \cos \left[\gamma G T\left(s_{i}-s_{j}\right)\right] \sin \left(D_{i j} t_{2}\right) \\
= & \frac{3}{8}\left(\frac{\hbar \omega}{k T}\right)^{2} \sum_{j=1}^{N}\left\{\left[I_{y j} \cos \left(2 \omega t_{1}\right)\right.\right. \\
+ & \left.I_{x j} \sin \left(2 \omega t_{1}\right)\right] \\
& \left.\times \cos \left[\gamma G T\left(s_{i}-s_{j}\right)\right] \sum_{i=1}^{N} \sin \left(D_{i j} t_{2}\right)\right\}
\end{aligned}
$$

Finally, we can quantify the term involving the dipolar couplings. Since we are free to choose the origin of our coordinate system, we can set $s_{i}=0$ and find

$$
\begin{aligned}
& \sum_{j=1}^{N} D_{i j} \cos \left(\gamma G T s_{j}\right) \\
& \approx \frac{-\mu_{0} \hbar \gamma^{2}}{16 \pi} \frac{N}{V} \int_{V} \frac{3 \cos ^{2} \vartheta-1}{r^{3}} \\
& \times \cos (\gamma G T s) r^{2} \sin \vartheta d r d \vartheta d \phi
\end{aligned}
$$

The approximation of the sum by an integral is valid if the distribution of spins can be considered continuous. The integral has a singularity at $r=0$, but in reality two spins will have a finite separation. If the volume of the sample is large enough, the integral converges as

$$
\begin{gathered}
\int_{r=r_{\min }}^{\infty} \int_{\vartheta=0}^{\pi} \int_{\phi=0}^{2 \pi} \frac{3 \cos ^{2} \vartheta-1}{r^{3}} \\
\times \cos (\gamma G T s) r^{2} \sin \vartheta d r d \vartheta d \phi \\
=-\frac{8 \pi}{3} \Delta_{s} \\
\Delta_{s} \equiv \frac{\left[3(s \cdot z)^{2}-1\right]}{2}
\end{gathered}
$$

The minimum separation distance $r_{\min }$ that is introduced as the lower limit of the integral is of course mathematically necessary to avoid the singularity at that point, but also physically reasonable, as two spins cannot be separated by a distance of 0 . The integral gives us the total effect of dipolar couplings on a spin at the center of an infinitely large sample. However, the effect is obviously distance dependent; therefore, let us only look at the polar part of this integral,

$$
\begin{aligned}
\int_{\vartheta=0}^{\pi} \int_{\varphi=0}^{2 \pi} \frac{3 \cos ^{2} \vartheta-1}{r^{3}} \cos (\gamma G T s) r^{2} \sin \vartheta d \vartheta d \phi \\
=2 \pi \times \gamma G T \int_{\vartheta=0}^{\pi} \frac{3 \cos ^{2} \vartheta-1}{\gamma G T r} \\
\quad \times \cos (\gamma G \operatorname{Tr} \cos \vartheta) \sin \vartheta d \vartheta
\end{aligned}
$$

This may be numerically solved as a function of $\gamma G \operatorname{Tr}$ (which is the interspin distance in units of gradient helix pitch). It then turns out that the bulk of dipolar interactions that become visible comes from pairs of spins by approximately one half helix pitch $(r / \gamma G T=\pi)$.

Following Eq. [40], the sum from Eq. [39] becomes

$$
\sum_{j=1}^{N} D_{i j} \approx \frac{N}{V} \frac{\mu_{0} \hbar \gamma^{2}}{6} \Delta_{s}
$$

It is now convenient to introduce a new variable, which we will call the 'dipolar demagnetizing time,' $\tau_{d}$. With the definition of

$$
\tau_{d} \equiv \frac{1}{\mu_{0} \gamma M_{0}}
$$


and considering that

$$
M_{0}=-\frac{N \hbar \gamma}{4 V}\left(\frac{\hbar \omega}{k T}\right)
$$

we find that

$$
\begin{aligned}
\rho= & \frac{3}{8}\left(\frac{\hbar \omega}{k T}\right)^{2} \\
& \times \sum_{i=1}^{N}\left[I_{x i} \sin \left(2 \Delta \omega t_{1}\right)+I_{y i} \cos \left(2 \Delta \omega t_{1}\right)\right] \\
& \times \frac{2}{3}\left(\frac{-t_{2} \Delta_{s}}{\tau_{d}}\right) \times \frac{k T}{\hbar \omega} \\
= & \frac{1}{4} \frac{\hbar \omega}{k T} \sum_{i=1}^{N}\left[I_{x i} \sin \left(2 \Delta \omega t_{1}\right)+I_{y i} \cos \left(2 \Delta \omega t_{1}\right)\right] \\
& \times\left(\frac{-t_{2} \Delta_{s}}{\tau_{d}}\right)
\end{aligned}
$$

Finally, the system evolves under the chemical shift as a function of $\left(\Delta \omega t_{2}\right)$ only. This signal behaves like a single-quantum transition in $t_{2}$, and like a double-quantum transition in $t_{1}$, in agreement with our experimental results! Interestingly, the signal increases during the acquisition time, unlike a regular FID, which decays during $t_{2}$. This reflects the action of the dipolar couplings, which transform two-spin one-quantum operators into magnetization during $t_{2}$. Of course, we have not taken relaxation into account, which competes with the action of the dipolar couplings and eventually leads to signal decay.

What is the magnitude of the signal? The analogous signal from a COSY sequence, using only the linear term of the density operator (Eq. [12]), is proportional to the Boltzmann factor only. Therefore, the ratio between the signal intensities is given by

$$
\frac{S_{\mathrm{CRAZED}}}{S_{\mathrm{COSY}}}=\frac{1}{4} \frac{t_{2} \Delta_{s}}{\tau_{d}}
$$

Let us quantify this result. The dipolar demagnetizing time, $\tau_{d}$, was defined previously in Eq. [43]. $M_{0}$, the equilibrium magnetization, is of course a function of the magnetic field strength, and so is the dipolar demagnetizing time. For a sample of water, the equilibrium magnetization is given by

$$
\begin{aligned}
M_{0}= & \frac{1}{4} \frac{N}{V}(\gamma \hbar)^{2} \frac{B_{0}}{k T} \\
= & \frac{1}{4} \frac{2 \times 6 \times 10^{23}}{18 \times 10^{-6} m^{3}} \\
& \times\left(2.68 \times 10^{8} \frac{1}{s T} \times 1.05 \times 10^{-34} J S\right)^{2} \\
& \times \frac{14.1 T}{1.38 \times 10^{-23} J K^{-1} \times 298 K} \\
= & 0.045 \frac{J}{T} \frac{1}{m^{3}}
\end{aligned}
$$

Therefore, the dipolar demagnetizing time in that case is

$$
\begin{aligned}
\tau_{d} & =\frac{1 A^{2} s T \times \mathrm{Tm}^{3}}{4 \pi \times 10^{-7} J \times 2.68 \times 10^{8} \times 0.045 J} \\
& =0.066 \mathrm{~s}
\end{aligned}
$$

This value is for a sample of pure water at 600 MHz. As we can see from the definition of $\tau_{d}$, this quantity is inversely proportional to both the field strength and the proton concentration in the sample.

Note that the derivation that we show in this paper is incomplete, because we terminate the expansion of the density operator after the quadratic term. Higher order terms in this expansion can become visible as well; for example, four-spin, single-quantum operators become magnetization after three successive dipolar couplings. The magnitude of the higher order term is not negligible. A complete derivation, as shown in (9), reveals that the FID really increases as $y=$ $J_{2}(x) / x$, where $x$ is $t_{2} \Delta / \tau_{d}$, and $J_{2}$ is the second order Bessel function. This function is graphed in Fig. 8. Note that the signal initially increases almost linearly with $t_{2}$-this is the limit in which the derivation in this paper is valid. Note that the maximum occurs when $x=2.2$. For a $z$ gradient, $\Delta s=1$, hence the maximum is at approximately $130 \mathrm{~ms}$ in our example.

Now let us consider the effects of relaxation. Transverse magnetization decays with a time constant $T_{2}^{*}$ (the "apparent transverse relaxation time"), which may be as long as $1 \mathrm{~s}$ in a wellshimmed sample, but may also be as short as a few tens of milliseconds, especially in vivo. This 


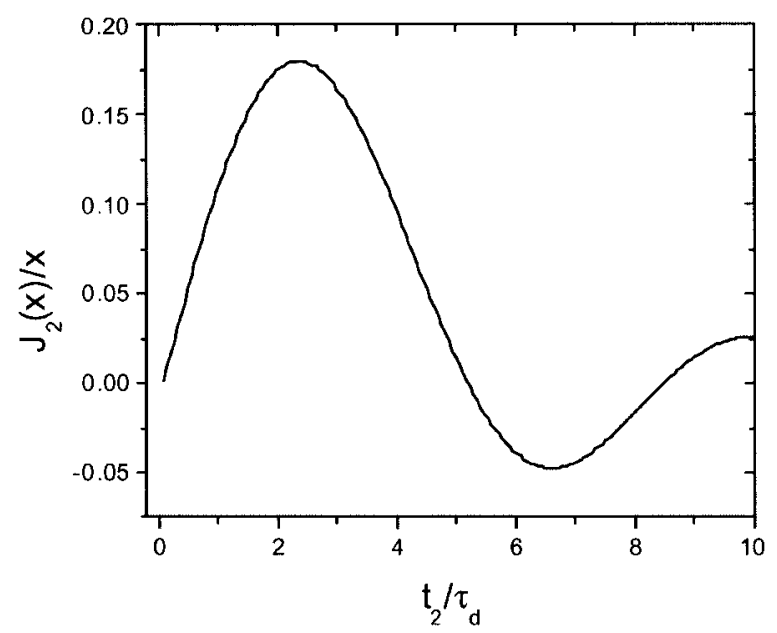

Figure 8 Theoretical shape of the CRAZED FID in the absence of relaxation.

limits the practicality of the iDQC method at lower field, where $\tau_{d}$ is long.

\subsection{Classical Description of the CRAZED Experiment}

As mentioned above, a parallel description of the CRAZED experiment is purely classical; that is, it only considers the evolution of magnetization under the action of RF pulses, the static magnetic field, and dipolar interactions from other spins. Classical description of the CRAZED sequence can be outlined as follows. During the $t_{1}$, the spins precess about the static magnetic field according to the conventional Bloch equations. This means, conceptually, that nothing but magnetization (i.e., nothing that could correspond to double-quantum coherences) evolves during that time. When gradient pulses are applied, dipolar couplings appear and lead to a nonlinear evolution (i.e., the evolution of the spins is dependent on the instantaneous state of the spin system). Quantitative analysis shows that the signal is exactly the same as the quantum treatment predicts; however, the apparent evolution at twice the resonance frequency during $t_{1}$ is merely an effect of the nonlinear evolution during the gradient pulses and $t_{2}$. This description is obviously conceptually different from the quantum description; a discussion of the truth of either of these descriptions is beyond the scope of this paper. However, as mentioned above, the predictive power of the quantum picture is extremely important for the design of novel pulse sequences using this effect.

\section{FIRST APPLICATIONS: HOMOGENIZED AND FMRI}

In the past few years, some applications of the CRAZED experiment have begun to emerge. In general, we are not restricted to intermolecular double-quantum coherences, as in the CRAZED experiment itself, but, in principle, any order of intermolecular multiple-quantum coherences may be achieved through the proper pulse sequence. Hence the general method is called 'intermolecular multiple-quantum coherence (iMQC) method.'

The strength of the iMQC method is that interactions and relationships between spins can be measured on a mesoscopic distance scale. As discussed above, the typical interaction distance that later becomes visible is on the order of $1 / 2$ pitch of the gradient helix, which can in practice be as short as $10 \mu \mathrm{m}$. This lower limit is approximate and essentially limited by the diffusion properties of the sample in relation to the length of the pulse sequence-if the helix pitch is to short, diffusion blurs the gradient helix so that it cannot refocus properly.

Most applications to date are based on intermolecular zero-quantum coherences (iZQCs). Since the coherence order is generally determined by the ratio of areas of the two gradient pulses, we need only a single gradient pulse to select for iZQCs. The crucial property of a zeroquantum coherence is that it evolves at the difference of the resonance frequency of the two spins. This means that the linewidth of an iZQC peak is a function not of the overall distribution of magnetic susceptibilities in the sample (the 'overall inhomogeneity'), but rather of the distribution of magnetic susceptibility gradients over the distance scale selected. Consider, for example, the HOMOGENIZED pulse sequence (16). This pulse sequence is identical to the CRAZED sequence, with three modifications: (1) The first gradient pulse is eliminated, for zero-quantum coherence selection; (2) the second RF pulse is a $45^{\circ}$ pulse - this achieves maximum signal, as a simple density operator calculation shows; and (3) at the end of the pulse sequence, a spin echo is added, in order to combat effects of relaxation.

The HOMOGENIZED pulse sequence then detects magnetization in $F_{2}$, and iZQCs in $F_{1}$. Consequently, when this pulse sequence is applied to a sample in an extremely inhomogeneous field, sharp lines can be achieved in $F_{1}$. In (16), it was shown that, in spite of a directly detected 
linewidth of several hundred $\mathrm{Hz}$, a triplet could be resolved in the indirectly detected dimension.

IMQC contrast may also prove invaluable in magnetic resonance imaging (MRI). Since it has been shown (17) that the contrast is fundamentally different from $T_{1}, T_{2}$, or $T_{2}^{*}$, and since there are good reasons to believe this contrast correlates with oxygenation, it may be very useful in diagnostic imaging. Another exciting application in this context is that to functional magnetic resonance imaging (fMRI) (18-21). In short, fMRI measures brain function through the relaxation properties of water protons in the brain. Upon neuronal activation, oxydative metabolism at the site of activation increases. Within a few seconds, blood flow increases as well and actually overcompensates for the increased oxygen demand. Hence oxygen concentration in the vicinity of the activated site increases. As a consequence, the relative concentration of (paramagnetic) deoxyhemoglobin decreases, and the apparent transverse relaxation time $T_{2}^{*}$ increases. Therefore, an image with $T_{2}^{*}$ weighted contrast shows increased intensity upon neuronal activation. This is called the BOLD ('blood oxygen level dependent') effect.

Since functional MRI measures susceptibility gradients related to brain activation, an iMQC method may have vastly improved sensitivity over the BOLD experiment. The signal change in the BOLD experiment is on the order of a few per cent. Considering the already staggering difficulties in taking images of a living organism in quick succession, it is obvious that we can only detect brain activation in the most fortunate circumstances. In the iMQC method, we have several variable parameters, most notably, the interaction distance between the two spins (which is the distance over which susceptibility gradients are measured). Even though the BOLD mechanism is not well understood at present, it is likely that the susceptibility gradients that change upon activation have a typical length, related to the vessel size. Therefore it is well possible that an iMQC experiment with appropriate parameters might detect a much larger signal change upon activation. In fact, it has recently been shown by our group (22) that functional activation in the visual cortex can be detected by the iMQC method; in this first experiment, we found that the iMQC activation map was much more focal than, and not fully congruent with, the BOLD activation map. Furthermore, the typical relative signal change upon activation was several times higher for the iMQC method. Future work will reveal if the iMQC method can indeed be routinely used for functional MRI.

\section{CONCLUSION}

The iMQC method was developed during the past decade from observations that seemed to contradict the very foundations of NMR. We know now that the signal is generated through a mechanism different than that in all other liquid state NMR experiments. This leads us to believe that a plethora of potential applications of this method exists; we have given here a glimpse into the two most exciting applications to date.

\section{REFERENCES}

1. Bloch F, Hansen WW, Packard ME. Nuclear induction. Phys Rev 1946; 69:127.

2. Lin Y-Y, Lisitza N, Ahn S, Warren WS. Science (in press).

3. He Q, Richter W, Vathyam S, Warren WS. Intermolecular multiple-quantum coherences and cross correlations in solution nuclear magnetic resonance. J Chem Phys 1993; 98:6779; Warren WS, Richter W, Hamilton Andreotti A, Farmer III BT. Generation of impossible cross peaks between bulk water and biomolecules in solution NMR. Science 1993; 262:2005.

4. Richter W, Lee S, Warren WS, He Q. Imaging with intermolecular multiple-quantum coherences in solution NMR. Science 1995; 267:654-657.

5. Deville G, Bernier M, Delrieux JM. Phys Rev B 1979; 19:5666.

6. Minot ED, Callaghan PT, Kaplan N. J Magn Reson 1999; 140:200-205.

7. Bowtell R, Bowley RM, Glover P. J Magn Reson 1990; 88:643-651.

8. Edzes HT. J Magn Reson 1990; 86:293-303.

9. Jeener J, Vlassenbroek A, Broekaert P. J Chem Phys 1995; 103:1309-1332.

10. Jeener J. NMR excitation with two pulses. Ampere International Summer School. Yugoslavia: Basko Polje; 1972.

11. Aue W, Bartholdi E, Ernst RR. Two-dimensional spectroscopy. Application to nuclear magnetic resonance. J Chem Phys 1976; 64:2229-2246.

12. Abragam A. The principles of nuclear magnetism. Oxford: Clarendon; 1961.

13. Ernst RR, Bodenhausen G, Wokaun A, Principles of nuclear magnetic resonance in one and two dimensions. Oxford: Clarendon; 1987. 
14. Lee S, Richter W, Vathyam S, Warren WS. Quantum treatment of the effects of dipole-dipole interactions in liquid nuclear magnetic resonance. $\mathrm{J}$ Chem Phys 1995; 105:874-900.

15. Harris RK. Nuclear magnetic resonance spectroscopy. London: Pitman; 1983.

16. Bandettini PA, Wong EC, Hinks RS, Tikofsky RS, Hyde JS. Time course EPI of human brain function during task activation. Magn Reson Med 1992; 25:390-397.

17. Warren WS, Ahn S, Mescher M, Garwood M, Ugurbil K, Richter W, Rizi RR, Hopkins J, Leigh JS. MR imaging contrast enhancement based on intermolecular zero quantum coherences. Science 1998; 281:247-251.

18. Vathyam S, Lee S, Warren WS. Homogeneous NMR spectra in inhomogeneous fields. Science 1996; 272:92-96.

19. Ogawa S, Menon RS, Kim S-G, Ugurbil K. On the characteristics of functional magnetic resonance imaging of the brain. Annu Rev Biophys Biomol Struct 1998; 27:447-474.

20. Kwong KK, Belliveau JW, Chesler DA, Goldberg IE, Weisskoff RM, Poncelet BP, Kennedy DN, Hoppel BE, Cohen MS, Turner R, Cheng H, Brady TJ, Rosen BR. Dynamic magnetic resonance imaging of human brain activity during primary sensory stimulation. Proc Natl Acad Sci USA 1992; 89:5675-5679.

21. Ogawa S, Tank DW, Menon R, Ellermann JM, Kim S-G, Merkle H, Ugurbil K. Intrinsic signal changes accompanying sensory stimulation: functional brain mapping with magnetic resonance imaging. Proc Natl Acad Sci USA 1992; 89:5951-5955.
22. Richter W, Richter M, Warren WS, Merkle H, Andersen P, Adriany G, Ugurbil K. Magn Reson Imag 2000; 18:489-494.

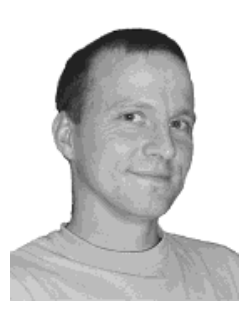

Wolfgang Richter was born and raised in Berlin, Germany. He received an M.S. in Chemistry at the University of Oklahoma in 1989 with Prof. Bing Fung, and a Ph.D. in Chemistry at Princeton University in 1995 with Prof. Warren Warren. He then did postdoctoral work at the Center for Magnetic Resonance Research at the University of Minnesota with Prof. Kamil Ugurbil. He is presently a staff scientist at the National Research Council's Institute for Biodiagnostics in Winnipeg. His current main research interest is functional magnetic resonance imaging of the human brain.

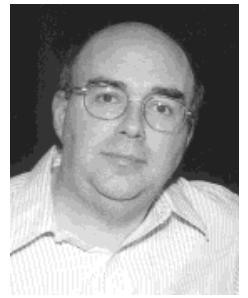

Warren S. Warren is a Professor of Chemistry at Princeton University, Director of the N.J. Center for Ultrafast Laser Applications, and Assoc. Director of the Princeton Center for Photonics and Optoelectronic Materials. He is also the editor of Advances in Magnetic Resonance. He received his bachelor's degree in 1977 from Harvard, and did his graduate work with Alex Pines at Berkeley, receiving the Ph.D. in 1980. He did postdoctoral work in laser spectroscopy with Ahmed Zewail at CalTech and moved to Princeton in 1982. Dr. Warren's research interests concentrate on the development and application of advanced pulsed techniques, principally in NMR and laser spectroscopy. 